\title{
Modeling, fabrication and high power optical characterization of plasmonic waveguides
}

\author{
Lavrinenko, Andrei; Lysenko, Oleg
}

Published in:

Proceedings of the 35th IEEE International Conference on Electronics and Nanotechnology International Conference on Electronics and Nanotechnology

Link to article, DOI:

10.1109/ELNANO.2015.7146884

Publication date:

2015

Document Version

Peer reviewed version

Link back to DTU Orbit

Citation $(A P A)$ :

Lavrinenko, A., \& Lysenko, O. (2015). Modeling, fabrication and high power optical characterization of plasmonic waveguides. In Proceedings of the 35th IEEE International Conference on Electronics and Nanotechnology International Conference on Electronics and Nanotechnology (pp. 245-248). IEEE. https://doi.org/10.1109/ELNANO.2015.7146884

\section{General rights}

Copyright and moral rights for the publications made accessible in the public portal are retained by the authors and/or other copyright owners and it is a condition of accessing publications that users recognise and abide by the legal requirements associated with these rights.

- Users may download and print one copy of any publication from the public portal for the purpose of private study or research.

- You may not further distribute the material or use it for any profit-making activity or commercial gain

- You may freely distribute the URL identifying the publication in the public portal 


\title{
Modeling, fabrication and high power optical characterization of plasmonic waveguides
}

\author{
O. Lysenko, A. Lavrinenko, \\ Department of Photonics Engineering \\ Technical University of Denmark \\ Kgs. Lyngby, Denmark \\ ollyse@fotonik.dtu.dk
}

\begin{abstract}
This paper describes modeling, fabrication and high power optical characterization of thin gold films embedded in silicon dioxide. The propagation vector of surface plasmon polaritons has been calculated within the effective index method for the wavelength range of $750-1700 \mathrm{~nm}$ and for the waveguide thicknesses of 15, 30 and $45 \mathrm{~nm}$. Fabrication process of such plasmonic waveguides with widths in the range of 1-100 $\mu \mathrm{m}$ and their quality inspection are described. Results of optical characterization of plasmonic waveguides using a high power laser with the peak power wavelength $1064 \mathrm{~nm}$ show significant deviation from the linear propagation regime of surface plasmon polaritons at the average input power of $100 \mathrm{~mW}$ and above. Possible reasons for this deviation are heating of the waveguides and subsequent changes in the coupling and propagation losses.
\end{abstract}

Keywords—nanophotonics; optical waveguides; plasmons

\section{INTRODUCTION}

The first experimental observation of surface plasmons in thin gold films embedded in silicon dioxide was published in 2000 [1]. A comprehensive study of long-range surface plasmon polaritons in thin gold films was performed by $\mathrm{P}$. Berini and co-workers and described in [2]. Optical characterization at the wavelength of $1550 \mathrm{~nm}$ showed good agreement between theoretical and experimental data. Effects of surface electromagnetic waves propagation in thin metal films can be used for development of integrated optics elements $[3,4,5]$. In some case, polymer benzocyclobutene is also used as a cladding of plasmonic waveguides instead of silicon dioxide. A review about surface plasmon polaritons is presented in [6].

In the present work, we describe modeling, fabrication and high power optical characterization of thin gold films embedded in silicon dioxide. The effective index method [7] was used for modeling plasmonic waveguides, and relevant calculations were performed using MATLAB. The modeling is described in section II. The fabrication of samples was performed in the university cleanroom and discussed in section III. The optical characterization of plasmonic waveguides was done using a high power laser with the peak power wavelength $1064 \mathrm{~nm}$. The experiment and obtained results are described in section IV.

\section{MODELING OF PLASMONIC WAVEGUIDES}

Modeling of thin gold films starts from the Maxwell equations, boundary conditions and calculation of dielectric permittivity of gold and silicon dioxide for the wavelength of interest [7,8].

The dielectric permittivity of silicon dioxide can be assumed constant for simplicity and equal to the following value at the wavelength $1064 \mathrm{~nm}$ :

$$
\varepsilon_{1}=2.12 \text {. }
$$

The dielectric permittivity of gold is calculated using the Drude model [7,8] and parameters from [7]:

$$
\begin{aligned}
& \varepsilon_{2}=\operatorname{Re}\left[\varepsilon_{2}\right]+i \cdot \operatorname{Im}\left[\varepsilon_{2}\right], \\
& \operatorname{Re}\left[\varepsilon_{2}\right]=1-\frac{\lambda^{2} \cdot \omega_{p}^{2} \tau^{2}}{\lambda^{2}+\tau^{2} \cdot 4 \pi^{2} c^{2}}, \\
& \operatorname{Im}\left[\varepsilon_{2}\right]=\frac{\omega_{p}{ }^{2} \tau \cdot \lambda^{3}}{2 \pi c \cdot \lambda^{2}+8 \pi^{3} c^{3} \tau^{2}} .
\end{aligned}
$$

Here, $\omega_{\mathrm{p}}=1.37 \cdot 10^{16} \mathrm{rad} / \mathrm{s}$ is the plasma frequency of gold, and $\tau=2.9 \cdot 10^{-14} \mathrm{~s}$ is the relaxation time of gold, $c$ is the speed of light in vacuum, and $\lambda$ is the wavelength of interest.

A thin gold film can be modeled as an infinitely wide slab with thickness $t$ and dielectric permittivity $\varepsilon_{2}$, embedded in medium with dielectric permittivity $\varepsilon_{1}$ (Fig. 1). Surface electromagnetic waves can propagate along the top and bottom boundaries of the slab and form coupled electromagnetic oscillations, one of which is called a long-range surface plasmon polariton. The propagation vector $\beta$ for the long-range mode of surface plasmon polaritons in this configuration can be found from the dispersion equation [8,9]:

$$
\tanh \left(\frac{t}{2} \cdot \sqrt{\beta^{2}-\varepsilon_{2} \frac{4 \pi^{2}}{\lambda^{2}}}\right)+\frac{\varepsilon_{2}}{\varepsilon_{1}} \cdot \frac{\sqrt{\beta^{2}-\varepsilon_{1} \frac{4 \pi^{2}}{\lambda^{2}}}}{\sqrt{\beta^{2}-\varepsilon_{2} \frac{4 \pi^{2}}{\lambda^{2}}}}=0
$$

This equation was solved numerically for $\beta$ in MATLAB for the thickness values of 15,30 and $45 \mathrm{~nm}$ and for the wavelength range of $750-1700 \mathrm{~nm}$. The real part of the propagation vector $\operatorname{Re}[\beta]$ is shown in Fig. 2. The calculation step is $50 \mathrm{~nm}$, and the obtained points are connected by straight lines. The real part of the propagation vector monotonically decays over the wavelength range and differs very little for selected values of the slab thickness. The obtained results show that the effective refractive index of plasmonic waveguides 
does not have significant dependence on the waveguide thickness for the wavelength range of interest.

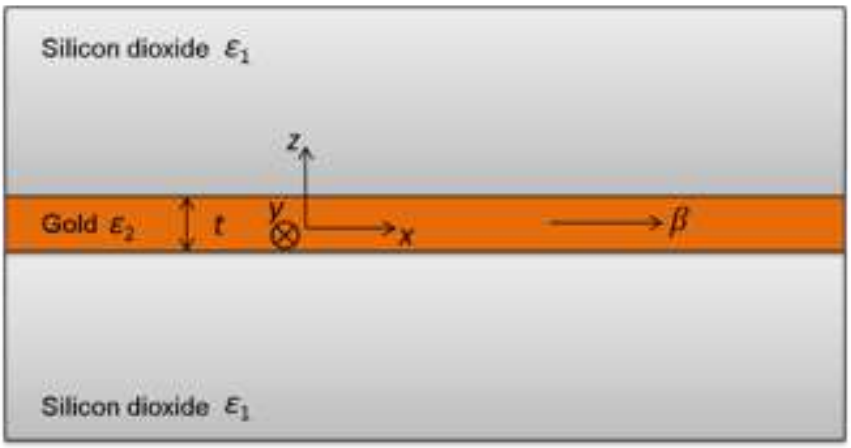

Fig.1. Thin gold film as a slab embedded in silicon dioxide

The imaginary part of the propagation vector $\operatorname{Im}[\beta]$ is shown on Fig. 3. The calculation step is $50 \mathrm{~nm}$, and the obtained points are connected by straight lines. The imaginary part of the propagation vector monotonically decays over the wavelength range, and differs significantly for selected values of the slab thickness. It is almost one order of magnitude smaller for the thickness of $15 \mathrm{~nm}$ than that for the thickness of $45 \mathrm{~nm}$. The imaginary part of $\beta$ is responsible for absorption and propagation losses in a plasmonic waveguide. Thinner plasmonic waveguides have less losses and, thus, longer propagation length of surface plasmon polaritons. The long range mode of surface plasmon polaritons can propagate over several millimeters and can be observed and studied experimentally for the selected thickness and wavelength range [7].

The obtained results and literature data [2,7,9] were used for the design and fabrication of plasmonic waveguide structures. The effective penetration depth for surface electromagnetic waves in gold is around 21-22 nm for the selected wavelength range and effective penetration depth in silicon dioxide is around the wavelength value. Thus, the gold film thickness should be around $50 \mathrm{~nm}$ or thinner and covered by several micrometers of dielectric from top and bottom side to observe the propagation of long-range surface plasmon polaritons.

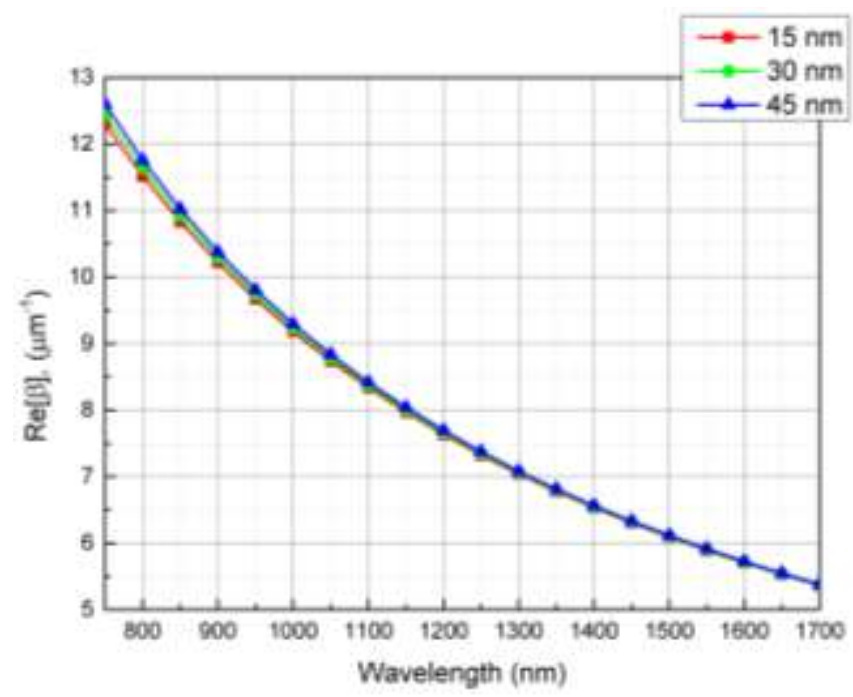

Fig.2. Real part of propagation vector as a function of wavelength

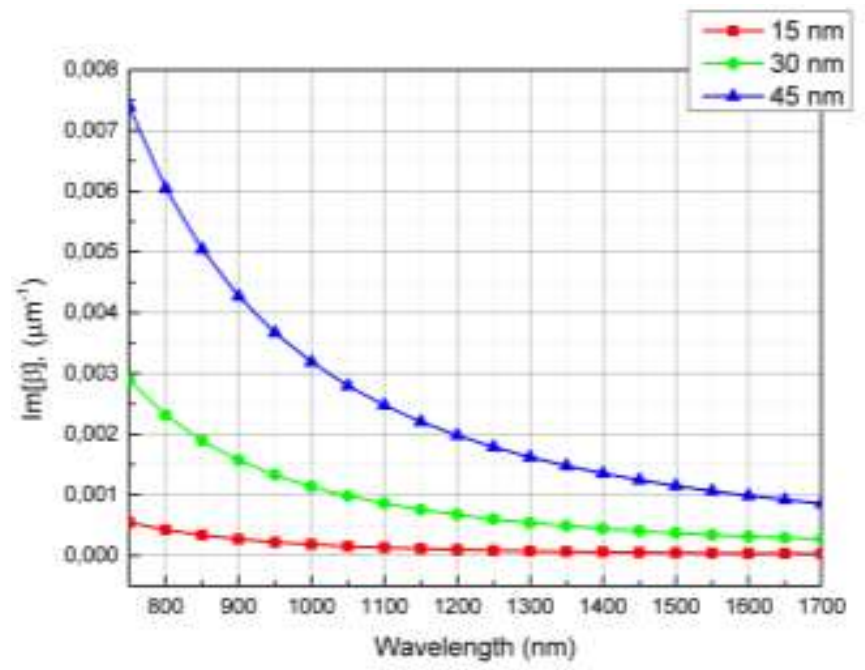

Fig.3. Imaginary part of propagation vector as a function of wavelength

\section{FABRICATION}

The prototype samples with plasmonic waveguides were fabricated in the university cleanroom. A photolithography mask was designed in CleWin layout editor and ordered from a commercial supplier. A silicon wafer with pre-made $6.5 \mu \mathrm{m}$ of the silicon dioxide cladding was used as a substrate. Standard ultraviolet lithography process was applied, and gold films were deposited on top of silicon dioxide afterwards using a sputtering machine. A chemical solution Piranha was used to remove the photoresist layer. A top cladding of silicon dioxide was deposited using the plasma-enhanced chemical vapor deposition method. The quality of plasmonic waveguides was checked using a scanning electron microscope. Image with several waveguides of different width obtained by the microscope is shown in Fig. 4. The waveguides are bright lines with widths $1,2,3,4,5,6$, and $7 \mu \mathrm{m}$. One fabricated wafer contained two groups of waveguides: thin waveguides and wide waveguides. Thin waveguides had width in the range of 1-10 $\mu \mathrm{m}$, with width step of $1 \mu \mathrm{m}$. Wide waveguides had width in the range of $10-100 \mu \mathrm{m}$, with width step of $10 \mu \mathrm{m}$. The distance between neighbor waveguides was $100 \mu \mathrm{m}$. This value is much bigger than typical penetration depth of surface electromagnetic waves in silicon dioxide, and each plasmonic waveguide could be investigated standalone, without influence on another neighbor waveguides. The difference between the nominal and fabricated widths of plasmonic waveguides was within the range of $1 \mu \mathrm{m}$. The thickness of waveguides was measured using a Dektak XT profilometer and was around 35 $\mathrm{nm}$. The thickness and refractive index of silicon dioxide were measured using a Filmtek 4000. The total thickness of silicon dioxide layer was around $11.5 \mu \mathrm{m}$, thus the thickness of deposited top cladding was around $5 \mu \mathrm{m}$. The refractive index of silicon dioxide was around 1.456 at $1064 \mathrm{~nm}$. The fabricated wafer was diced, and 15 rectangular slices with plasmonic waveguides of different width and length were collected in the chessboard (Fig. 5). The width of rectangular slices was 1.5 $\mathrm{cm}$, and the length was 2, 3, 4, 5 and $6 \mathrm{~mm}$. The top raw contains slices with 5 identical groups of wide plasmonic 
waveguides, each group has waveguides with the width in the range of 10-100 $\mu \mathrm{m}$ and the groups are separated

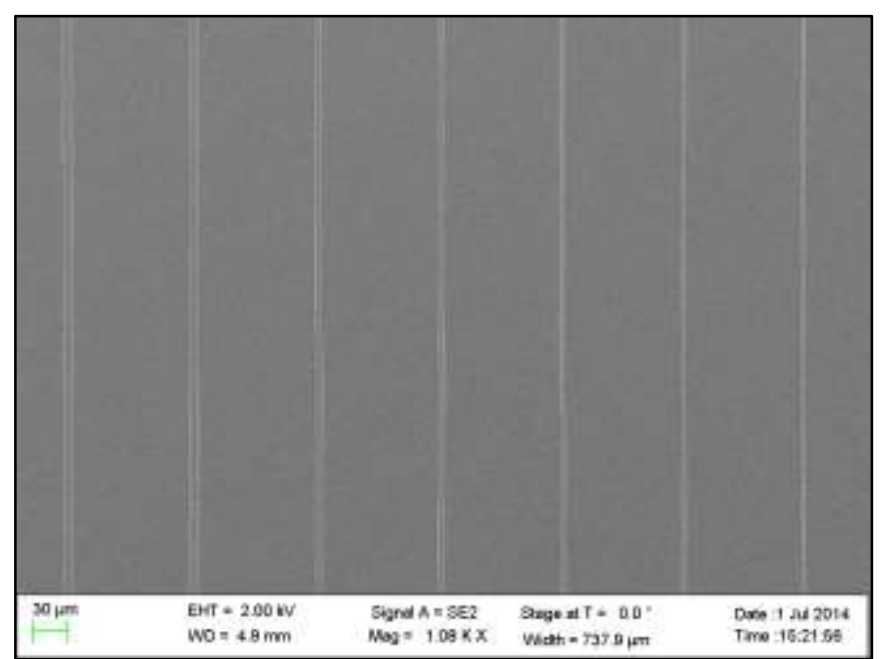

Fig.4. Scanning electron microscope image of plasmonic waveguides

by the distance of $500 \mu \mathrm{m}$. The total number of waveguides in each slice is $5 \times 10=50$. The middle raw contains slices with 10 identical groups of thin waveguides, each group has waveguides with the widths of $1-10 \mu \mathrm{m}$ and the groups are separated by the distance of $150 \mu \mathrm{m}$. The total number of waveguides in each slice was $10 \times 10=100$.

The bottom raw contains slices with a thin gold film covering the whole surface of slices. The rectangular samples from the top raw containing wide waveguides were selected for optical characterization.

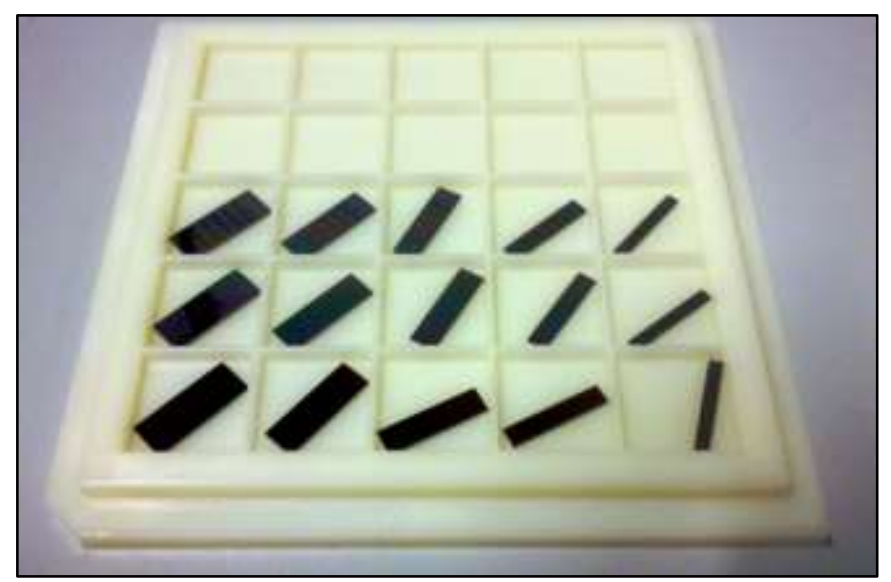

Fig.5. Chessboard with fabricated samples

\section{EXPERIMENT}

The experimental setup to characterize the samples is shown on Fig. 6. The laser source is picosecond SuperK EXTREME (NKT Photonics) with the maximum average power $15 \mathrm{~W}$, repetition rate $78 \mathrm{MHz}$ and peak power wavelength $1064 \mathrm{~nm}$. A laser beam propagated in the stepindex optical fiber to the fiber port, passed polarizers and coupled again to the polarization maintaining fiber. Two linear polarizers are used to tune the beam power without changing the laser operational current, and to keep the fixed linear polarization of output light. The first polarizer rotated to tune its polarization direction with respect to the second polarizer direction, and, thus, the beam power was tuned from $0 \mathrm{~W}$ to

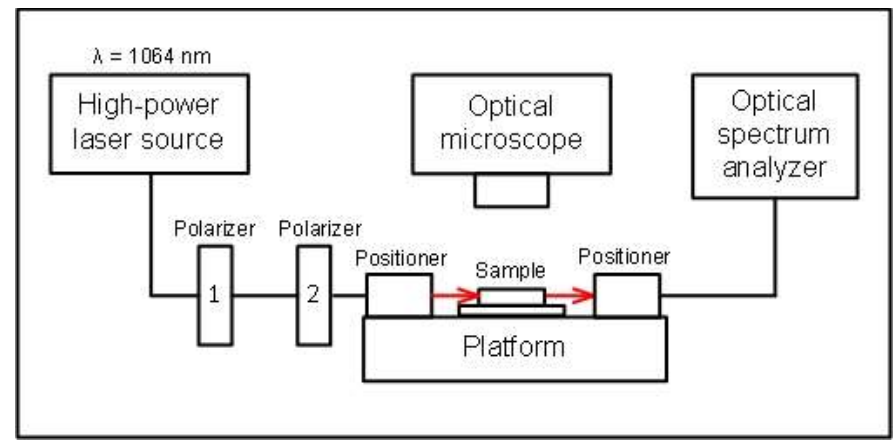

Fig.6. Experimental setup for high-power optical characterization

a half of the maximum value. The output beam polarization is maintained to match the transverse magnetic mode of surface plasmon polaritons in waveguides. The samples were placed by one on the moving platform and two positioners were used to implement the end-fire light coupling to plasmonic waveguides. A single mode fiber is used to collect and deliver the transmitted flux to the optical spectrum analyzer AQ6315E (Yokogawa). The samples motion and fibers alignment are controlled by the optical microscope Axiotech vario (Carl Zeiss). The polarization maintaining fiber is a photonic crystal fiber with the core diameter $10 \mu \mathrm{m}$. The single mode fiber is a photonic crystal fiber with the core diameter $10 \mu \mathrm{m}$. Plasmonic waveguides with the width of $10 \mu \mathrm{m}$ were selected for investigation to have the most coupling efficiency. Results of transmission measurement for plasmonic waveguides of different lengths and at the average power in the range below 1 $\mathrm{mW}$ were reported in [10]. The new results of high power optical characterization are shown on Fig. 7.

The horizontal axis corresponds to the laser reference power measured without sample by the fiber to fiber direct coupling method. The polarization maintaining fiber was placed in front of the single mode fiber, and transmitted power was measured by the optical spectrum analyzer. Thus, the first polarizer was calibrated according to its polarization direction and values of transmitted power. The maximum possible power used in the experiment was limited by damage threshold of the first polarizer and also by coupling efficiency of the 


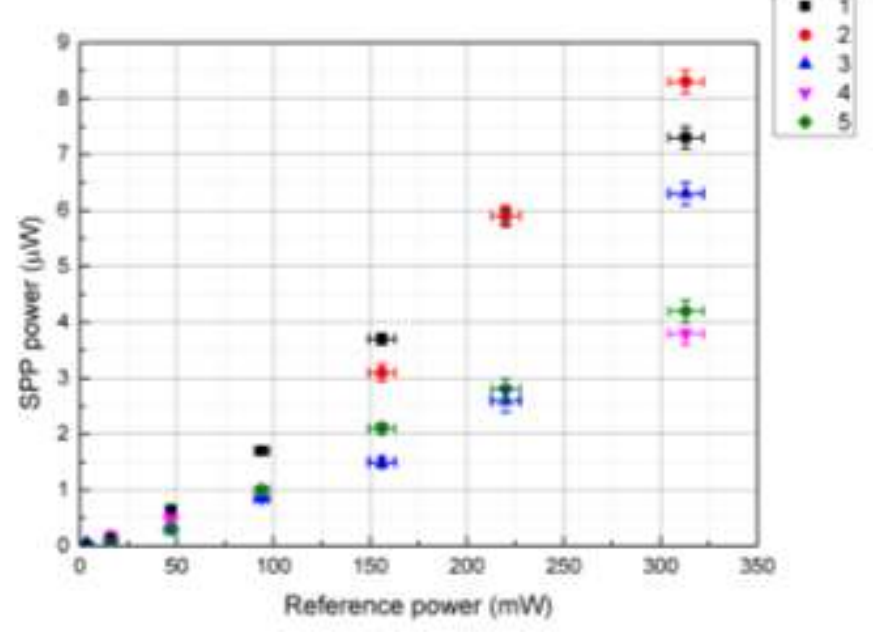

Fig.7. Power out of plasmonic waveguides as a function of reference power

polarization maintaining fiber. The reference power was tuned in the range from 0 to about $320 \mathrm{~mW}$. The horizontal error bars include calibration error of the first polarizer and statistical error of the optical spectrum analyzer. Ten consequent measurements were performed to obtain each point of the reference power. The vertical axis shows transmitted power though five plasmonic waveguides with the length $3 \mathrm{~mm}$ and width $10 \mu \mathrm{m}$. The vertical error bars include calibration error of the first polarizer and statistical error of the optical spectrum analyzer. Ten consequent measurements were performed to obtain each point of the transmitted power. The experimental data show monotonic increase of transmitted power with increase of the reference power. However, the trend for each plasmonic waveguide is somewhat different from expected linear dependence. The waveguides were nominally identical, but were situated in different places within one rectangular slice, and had different end-fire coupling efficiency with the optical fibers. The difference in coupling efficiency could be one of the possible reasons to explain trend deviations. The transmitted power discrepancy between plasmonic waveguides becomes even higher as the reference power increases, thus a power dependence of the coupling losses is observed. The second possible reason of the unusual power trends is propagation losses in plasmonic waveguides. The light propagating in plasmonic waveguides is absorbed in gold and silicon dioxide and, for high power regime, heating of waveguides can effectively change the optical parameters of materials. As the reference power increases, the change in propagation losses becomes significant and results in deviation from the linear trend for individual waveguides. The obtained power trends show that this deviation from the linear propagation regime becomes important for the reference power values in the range of $100 \mathrm{~mW}$ and above. For the reference power values in the range below $100 \mathrm{~mW}$, the propagation regime in plasmonic waveguides can be assumed linear and the power discrepancy between waveguides is mainly due to different end-fire coupling efficiency.
We have described modeling, fabrication and high power optical characterization of plasmonic waveguides based on thin gold films embedded in silicon dioxide. The effective index method was applied to calculate the propagation vector of long-range surface plasmon polaritons. The real and imaginary parts of the calculated propagation vector monotonically decreases as the light wavelength increases. The real part of the propagation vector $\operatorname{Re}[\beta]$ does not change significantly as the thickness of plasmonic waveguides changes from $15 \mathrm{~nm}$ to 45 $\mathrm{nm}$, and the imaginary part of the propagation vector $\operatorname{Im}[\beta]$ increases almost by one order of magnitude as the thickness of plasmonic waveguides changes from $15 \mathrm{~nm}$ to $45 \mathrm{~nm}$.

Fabrication process of the samples with plasmonic waveguides was described in detail and the quality inspection of waveguides by the scanning electron microscope was discussed. The fabrication error for the width of plasmonic waveguides was within the range of $1 \mu \mathrm{m}$.

High power optical characterization of plasmonic waveguides with the width $10 \mu \mathrm{m}$, length $3 \mathrm{~mm}$, and thickness $35 \mathrm{~nm}$ was performed. The obtained power trends for five waveguides of the same nominal geometry showed a deviation from the linear propagation regime for values of the reference power in the range of $100 \mathrm{~mW}$ and above. The differences in coupling loss and heating of waveguides by a high power laser beam could be possible reasons of the power deviation. The quantitative analysis of this phenomenon will be the subject of the further research in this direction.

\section{REFERENCES}

[1] R. Charbonneau, P. Berini, E. Berolo and E. Lisicka-Shrzek, "Experimental observation of plasmon-polariton waves supported by a thin metal film of finite width," Opt. Lett., vol. 25, no. 11, pp. 844-846, June 2000.

[2] P. Berini, R. Charbonneau, N. Lahoud and G. Mattiussi, "Characterization of long-range surface-plasmon-polariton waveguides," J. Applied Physics, vol. 98, 043109, August 2005.

[3] A.Boltasseva, T. Nikolajsen, K. Leosson, K. Kjaer, M. Larsen and S. Bozhevolnyi, "Integrated optical components utilizing long-range surface plasmon polaritons," J. Lightwave Technology, vol. 23, no. 1, pp. 413-422, January 2005.

[4] A. Boltasseva, S. Bozhevolnyi, "Directional couplers using long-range surface plasmon polariton waveguides," IEEE J. Selected Topics in Quantum Electronics, vol. 12, no. 6, pp. 1233-1241, November/December 2006.

[5] R. Charbonneau, C. Scales, I. Breukelaar, G. Mattiussi and P. Berini, "Passive integrated optics elements based on long-range surface plasmon polaritons," J. Lightwave Technology, v. 24, no. 1, pp. 477494, January 2006.

[6] P. Berini, "Long-range surface plasmon polaritons", Advances in Optics and Photonics, vol. 1, issue 3, pp. 484-588, September 2009.

[7] M. Zalkovskij, Surface plasmons in nanostructures, M.S. thesis, pp. 1134, University of Copenhangen, January 2009.

[8] S. Maier, Plasmonics: fundamentals and applications, pp. 21-34, Springer, New York, May 2007.

[9] V. Klimov, Nanoplasmonics, pp. 63-85, Pan Stanford, July 2014.

[10] M. Bache, A. Lavrinenko, O. Lysenko and R. Malureanu, "Nanoplasmonic solution for nonlinear optics", DTU Sustain Conference, Technical University of Denmark, December 2014.

\section{CONCLUSION}

